\title{
Maternal psychosocial risk factors and child gestational epigenetic age in a South African birth cohort study
}

\author{
Nastassja Koen (iD ${ }^{1,2,3^{凶}}$, Meaghan J. Jones (D) $^{4}$, Raymond T. Nhapi ${ }^{5,6}$, Marilyn T. Lake ${ }^{5}$, Kirsten A. Donald (iD ${ }^{3,7}$, Whitney Barnett ${ }^{8}$, \\ Nadia Hoffman ${ }^{1}$, Julia L. Maclsaac ${ }^{9}$, Alexander M. Morin ${ }^{9}$, David T. S. Lin ${ }^{9}{ }^{9}$, Michael S. Kobor ${ }^{9,10,11}$, Karestan C. Koenen ${ }^{12,13}$, \\ Heather J. $\operatorname{Zar}^{8}$ and Dan J. Stein (D) ${ }^{1,2,3}$
}

(c) The Author(s) 2021

\begin{abstract}
Accelerated epigenetic aging relative to chronological age has been found to be associated with higher risk of mortality in adults. However, little is known about whether and how in utero exposures might shape child gestational epigenetic age (EA) at birth. We aimed to explore associations between maternal psychosocial risk factors and deviation in child gestational EA at birth (i.e., greater or lower EA relative to chronological age) in a South African birth cohort study-the Drakenstein Child Health Study. Maternal psychosocial risk factors included trauma/stressor exposure; posttraumatic stress disorder (PTSD); depression; psychological distress; and alcohol/tobacco use. Child gestational EA at birth was calculated using an epigenetic clock previously devised for neonates; and gestational EA deviation was calculated as the residuals of the linear model between EA and chronological gestational age. Bivariate linear regression was then used to explore unadjusted associations between maternal/child risk factors and child gestational EA residuals at birth. Thereafter, a multivariable regression method was used to determine adjusted associations. Data from 271 maternal-child dyads were included in the current analysis. In the multivariable regression model, maternal PTSD was significantly and negatively associated with child gestational EA residuals at birth $(\beta=-1.95 ; p=0.018)$, controlling for study site, sex of the child, head circumference at birth, birthweight, mode of delivery, maternal estimated household income, body mass index (BMI) at enrolment, HIV status, anaemia, psychological distress, and prenatal tobacco or alcohol use. Given the novelty of this preliminary finding, and its potential translational relevance, further studies to delineate underlying biological pathways and to explore clinical implications of EA deviation are warranted.
\end{abstract}

Translational Psychiatry (2021)11:358; https://doi.org/10.1038/s41398-021-01434-3

\section{INTRODUCTION}

Maternal exposure to psychosocial risk factors during pregnancy -including traumatic stressors, psychiatric disorders and symptoms, and substance misuse - constitute a notable public health concern; and may be associated with poor birth outcomes and adverse health and development in affected children [1-3]. Given the high burden of psychosocial risk factors in low- and middleincome countries (LMICs) such as South Africa [4], maternal-child dyads in these settings are particularly vulnerable. Prenatal psychosocial risk exposure may be associated with measurable changes in DNA methylation (DNAm) at specific genomic sites in the affected children [5]. It is noteworthy, though, that a recent meta-analysis found no large, epigenome-wide effects of prenatal maternal stress on child differential DNAm [6]. Recent work has also investigated DNAm as a predictor of chronological age in children and adults $[7,8]$. However, given the limitations in transferability of such 'epigenetic clocks' (which were calibrated initially for adult populations); specialised clocks have recently been generated to estimate gestational age at birth [9-11].

Accelerated epigenetic aging-i.e., greater DNAm gestational age relative to chronological age-has been found to be associated with increased risk of mortality in adults [12-14] (and potentially with increased risk of age-related disorders [14]). Further, recent work has reported significant associations between a number of maternal-child biomedical factors (e.g., prepregnancy maternal obesity; maternal age over 40 years at

\footnotetext{
${ }^{1}$ Department of Psychiatry and Mental Health, University of Cape Town, Cape Town, South Africa. ${ }^{2}$ South African Medical Research Council (SAMRC) Unit on Risk and Resilience in Mental Disorders, Cape Town, South Africa. ${ }^{3}$ Neuroscience Institute, University of Cape Town, Cape Town, South Africa. ${ }^{4}$ Biochemistry and Medical Genetics, Max Rady College of Medicine, Rady Faculty of Health Sciences, University of Manitoba, Children's Hospital Research Institute of Manitoba, Winnipeg, Manitoba, Canada. ${ }^{5}$ Department of Paediatrics and Child Health, Red Cross War Memorial Children's Hospital, University of Cape Town, Cape Town, South Africa. ${ }^{6}$ Division of Epidemiology and Biostatistics, School of Public Health and Family Medicine, University of Cape Town, Cape Town, South Africa. ${ }^{7}$ Division of Developmental Paediatrics, Department of Paediatrics and Child Health, Red Cross War Memorial Children's Hospital, University of Cape Town, Cape Town, South Africa. ${ }^{8}$ Department of Paediatrics \& Child Health and SAMRC Unit on Child and Adolescent Health, University of Cape Town, Cape Town, South Africa. ${ }^{9}$ Department of Medical Genetics, Centre for Molecular Medicine and Therapeutics, BC Children's Hospital Research Institute, University of British Columbia, Vancouver, Canada. ${ }^{10} \mathrm{BC}$ Children's Hospital Research Institute, Vancouver, Canada. ${ }^{11}$ Canadian Institute for Advanced Research, Toronto, Canada. ${ }^{12}$ Department of Epidemiology, Harvard T.H. Chan School of Public Health, Boston, MA, USA. ${ }^{13}$ Psychiatric and Neurodevelopmental Genetics Unit and Department of Psychiatry, Massachusetts General Hospital, Boston, MA, USA. ${ }^{\circledR}$ email: nastassja.koen@uct.ac.za
} 
delivery; lower $1 \mathrm{~min}$ Apgar score) $[15,16]$ and accelerated epigenetic aging in the affected children. Of note, associations with lower relative epigenetic age (EA) have also been reported (e.g., in the case of prior insulin-treated gestational diabetes mellitus [15]). Further, there is emerging interest in the potential effects of psychosocial risk factors-for example, maternal depression [17], and greater infant distress (in the context of low caregiver contact) [18] have each been found to be associated with lower EA relative to chronological age. However, there remains a relative paucity of existing literature in this field, particularly in LMICs.

This analysis was nested within the Drakenstein Child Health Study (DCHS), an ongoing, interdisciplinary, longitudinal birth cohort study investigating determinants of maternal-child health in a poor, peri-urban South African community [19-22]. We have previously reported a high burden of maternal psychosocial risk factors (e.g., traumatic stress exposure/posttraumatic stress disorder (PTSD), alcohol and tobacco use); and significant associations between these factors and adverse birth and neurodevelopmental outcomes in the affected children in this cohort [22-27]. In the current analysis, we aimed to investigate associations between maternal psychosocial risk factors and deviation in child gestational EA at birth, i.e., greater or lower EA relative to chronological age. We hypothesised that key maternal risk factors would be significantly associated with our outcome of interest in this study sample.

\section{MATERIALS/SUBJECTS AND METHODS \\ Participants}

Pregnant women were recruited at 20-28 weeks' gestation from two primary care clinics in the Drakenstein sub-district in Paarl, Western Cape, South Africa-one (TC Newman) serves a predominantly mixed ancestry community; while the other (Mbekweni) serves primarily a black African ancestry community. Within the DCHS, mothers are followed throughout pregnancy and childbirth until the index child is at least 5 years old [19-22]. General inclusion and exclusion criteria for the DCHS are described fully elsewhere [20].

For this analysis, samples of mothers with complete and accurate sociodemographic, biomedical and psychosocial phenotype data; with recorded maternal informed consent; and with non-contaminated Illumina Infinium HumanMethylation450K or MethylationEPIC BeadChip umbilical cord blood data were eligible for inclusion [28] (see also Statistical Analyses, below).

This study sample included mothers enroled into the larger study between March 2012 and March 2015. From a total of 1225 mothers enroled, 1143 were retained in the cohort and delivered live births. From this sub-sample, 275 dyads had noncontaminated HumanMethylation450K (Batch 1, $n=120$ ) or MethylationEPIC (Batch 2, $n=155$ ) BeadChip cord blood data [28]; and available maternal and child phenotype data. This subsample was initially selected for analysis based on a number of risk criteria of relevance to the larger DCHS. Of note, cord blood samples analysed using the EPIC BeadChip (Batch 2) were selected primarily to optimise those with prenatal maternal trauma exposure and/or PTSD. Two sets of twins were excluded, due to concerns of analytic mixed effects. Thus, data from a sub-sample of 271 maternal-child dyads were included in the current analysis.

\section{Variables and measurement}

Maternal sociodemographic characteristics; antenatal biomedical and psychosocial risk factors; and anthropometric outcomes at birth were assessed, as is detailed below. Maternal psychosocial assessment in the DCHS has been described fully previously [20].

Maternal sociodemographic characteristics. Socioeconomic status (SES) and related sociodemographic characteristics were assessed using a questionnaire adapted from the South Africa Stress and Health Study (SASH) [29].

Maternal biomedical risk factors. For our purposes, maternal Human Immunodeficiency Virus (HIV) status was assessed by rapid testing at enrolment if the mother's HIV status was unknown at that time. Pre-eclampsia was defined as new-onset hypertension after 20 weeks' gestation with proteinuria or other organ dysfunction; and maternal anaemia was conservatively defined as any haemoglobin measurement $<10 \mathrm{~g} / \mathrm{dl}$ [22].

Maternal antenatal psychosocial risk factors. Exposure to stressful events-either recently or in one's lifetime-was assessed using the modified World Mental Health Life Events Questionnaire (LEQ; adapted for our purposes from the SASH [29]); the Childhood Trauma Questionnaire (CTQ) [30]; and the Intimate Partner Violence (IPV) Questionnaire (adapted from the World Health Organization (WHO) multi-country study on women's health and domestic violence against women [31]. The modified PTSD Symptom Scale (mPSS) [32, 33] was used as a rapid screening for PTSD, based on the DSM diagnostic criteria (with additional introductory items devised for our purposes to assess for the presence of a DSM-defined psychological trauma). The mPSS screens for PTSD symptoms in the preceding 2 weeks. In addition - for the purposes of the DCHS-we generated a composite measure of maternal lifetime trauma exposure (as assessed antenatally), incorporating data from the LEQ, CTQ, IPV questionnaire, and mPSS; as well as from the clinician-administered MINI (Mini International Neuropsychiatric Interview) [34-36]. Psychological distress was assessed using the Self-Reporting Questionnaire (SRQ-20) [37-39]; and depression using the Beck Depression Inventory II (BDI-II) [40-42] and the Edinburgh Postnatal Depression Scale (EPDS) [43, 44]. Maternal substance use $^{1}$ was identified using the WHO Alcohol, Smoking, and Substance Involvement Screening Test (ASSIST) [45-47] and a retrospective Alcohol Exposure Questionnaire (AEQ). The AEQ was designed for the purposes of the DCHS to quantify alcohol use (during any of the three trimesters of pregnancy) in mothers assessed as high-risk on the ASSIST.

Anthropometry at birth. Newborn weight and head circumference at birth were measured by trained clinical staff, and the relevant $z$ scores were then calculated using the Fenton preterm growth charts [48, 49]. Following the WHO convention, low weight-for-age $z$ score (WAZ) and low head circumference-for-age $z$ score (HCAZ) each was defined as a score of 2 standard deviations or more below the mean [50]. Prematurity was defined as birth before 37 completed weeks' gestation, as estimated by antenatal ultrasound measurement (for the majority of participants). In the rare case of missing or implausible data, fundal height and then maternal recall of last menstrual period were used to determine gestation at birth. Low birthweight was defined as less than $2500 \mathrm{~g}$.

Umbilical cord blood collection. In the DCHS, umbilical cord blood was collected by trained staff after newborn delivery but before delivery of the placenta. The cord was clamped and cut, after which the clamp was released and cord blood drained by gravity into a kidney dish. Thereafter, cord blood was collected using a syringe for processing and storage [28]. Staff were specifically trained to ensure only cord blood drained into the collection dish and that to their best ability, no other/external blood was collected. Further, cord blood samples previously identified as being considerably contaminated with maternal blood (presumably resulting from mixing

${ }^{1}$ Prenatal tobacco use derived from ASSIST item assessing recent frequency of use. 
of blood during sample collection) [28], were excluded from the current analysis.

\section{Ethical considerations}

The DCHS was approved by the Human Research Ethics Committee (HREC) of the Faculty of Health Sciences, University of Cape Town (UCT) and by the Western Cape Provincial Research Committee. Within the DCHS, all potential participants were provided with a consent form which included a description of the scope and aims of the study, in their preferred language (English, Afrikaans, or isiXhosa). Following verbal and written informed consent, mothers were asked to complete a battery of self-report measures, and to respond to select clinician-administered assessment tools (e.g., the MINI) at an antenatal study visit (28-32 weeks' gestation). While maternal phenotype data from a number of postnatal timepoints are also collected within the larger DCHS $[19,20]$; the current analysis pertains to the antenatal maternal assessment only.

All measures were administered by trained study staff in either English, Afrikaans or isiXhosa, as per participant preference. Interviews were conducted in private onsite consultation rooms and every effort was made to ensure confidentiality. Participants were also provided with standard reimbursement for transport costs. On completion of the study assessment, participants with suspected psychopathology or psychosocial risk were referred by study staff to appropriate care providers in the community, according to a standard operating procedure devised for the DCHS. Health promotion information leaflets and bookmarks designed by the study team (and translated into participants' preferred language) were also offered to all enroled mothers, and included contact details for local and accessible health service providers in addition to the formal referral processes [25].

\section{Statistical methods}

Data were analysed using R [51] and STATA [52].

Maternal-child phenotype. Frequency distributions and medians (interquartile ranges) were used to describe maternal sociodemographic characteristics; biomedical and psychosocial risk profiles; as well as infant anthropometric parameters at birth.

Gestational epigenetic age at birth. As has been described fully previously [28], cord blood DNAm was measured using the Illumina Infinium HumanMethylation450K (Illumina, San Diego, USA) or the MethylationEPIC BeadChip [53] as per manufacturers' instructions. Raw data were imported into Illumina GenomeStudio Software for background subtraction and colour correction, and then exported for processing using the lumi package in $\mathrm{R}$ (version 3.2.3). Quality control procedures were standardised and are discussed in detail elsewhere $[28,54]$. In summary, CpGs with high detection $p$ values (>0.05), those on the $X$ or $Y$ chromosome, and those with prior poor performance were removed. Normalisation was undertaken using subset quantile within array (SWAN) for the $450 \mathrm{~K}$ data; or beta mixture quantile (BMIQ) for the EPIC data. Finally, ComBat was used to correct for chip (all data) and row effects (450K data only).

Gestational EA at birth was calculated using an epigenetic clock particularly designed for newborns which outputs gestational age [11] and is based on prior work in adult populations using chronological age [7]. This gestational age clock [11] was trained on 1068 samples (mean ultrasound estimated gestational age 279.6 days with $95 \% \mathrm{Cl} 279-280.3$ days) and tested on 685 samples (mean ultrasound estimated gestational age 279.4 days with $95 \%$ Cl 278.5-280.2 days), all from the Norwegian Mother and Child Cohort Study (MoBa) study $[55,56]$. From 5,474 CpGs associated with ultrasound-estimated gestational age, the final elastic net regression predictor contained $96 \mathrm{CpGs}, 8$ of which are missing on the EPIC array. For samples in this study generated using the EPIC data, the 8 missing CpGs were set to the mean values of the $450 \mathrm{~K}$ samples. In the current analysis, gestational EA deviation was calculated as the residuals of the linear model between gestational EA and chronological gestational age [57], stratified by batch.

Associations between maternal psychosocial risk factors and gestational epigenetic age deviation at birth. Bivariate analyses were used to explore unadjusted associations between key sociodemographic, biomedical and psychosocial variables, Supplementary Table A, and child gestational EA deviation at birth. Thereafter, a multivariable regression approach was used to determine adjusted associations. The multivariable regression model was obtained by retaining variables with at least a trendlevel difference $(p<0.1)$ at the bivariate level (in attempts to minimize Type 2 error); and those that theoretically were suspected or hypothesized to be significantly associated with the outcome of interest. Stratification of the regression model by batch/methylation microarray (i.e., Infinium HumanMethylation450K versus MethylationEPIC BeadChip) was not undertaken; as samples analysed using the EPIC BeadChip were selected based primarily on trauma/PTSD phenotype. Given that this was not the case for samples analysed using the $450 \mathrm{~K}$ chip, selection criterion was a potential confounder. Further, EA was found not to differ significantly between batches in exploratory t-test analyses. Nonetheless, potential batch effects were taken into account by stratifying the primary outcome computations-EA residuals-by batch (as described above).

\section{RESULTS}

Maternal sociodemographic and biomedical characteristics

This study sample comprised 151 (56\%) women recruited from Mbekweni and 120 (44\%) from TC Newman. At enrolment, the median (IQR) age of mothers was approximately $26(22 ; 31)$ years, Table 1. Approximately a third of mothers were primigravid, and most $(61 \%)$ were neither married nor co-habiting with a partner. Despite approximately half of the study sample having completed some secondary education, nearly three quarters (73\%) were unemployed and the vast majority (83\%) reported a monthly household income of $\leq$ ZAR 5000 ( $\approx$ USD 350). The prevalence of maternal HIV infection in the study sample was $24 \%$, which is representative of the prevalence (21\%) in the larger DCHS cohort [22]. A statistically significant site-specific difference in maternal HIV infection was also noted $(p<0.01)$, with a significantly higher prevalence at Mbekweni, than at TC Newman. No child in this study sample was HIV infected.

\section{Maternal antenatal psychosocial risk}

Most mothers (63\%) in this study sample reported exposure to any psychological trauma during their lifetimes, as assessed using the mPSS and LEQ. When applying our composite trauma exposure variable, this prevalence was found to increase to $88 \%$, Table 1 . Almost half $(42 \%)$ of the study sample had been exposed to maltreatment (abuse/neglect) during childhood; and the prevalence of lifetime exposure to intimate partner violence (IPV) was $54 \%$ (with $42 \%$ having experienced IPV during the past year). The prevalence of current PTSD was 30\%, with significantly more mothers at Mbekweni than TC Newman affected.

Despite a relatively low median (IQR) score on the Life Events Questionnaire $[2(1 ; 3)], 29 \%$ of the study sample reported symptoms of psychological distress on the SRQ-20. Similarly, a quarter scored above threshold for depression on the BDI-II (25\%) (and almost a third on the EPDS (30\%)). Alcohol consumption during pregnancy was reported in $17 \%$ of participants, and prenatal tobacco use in $29 \%$. A notable site-specific difference was evident, with the prevalence of tobacco use significantly higher among mothers from TC Newman. 
Table 1. Maternal sociodemographic characteristics and antenatal psychosocial risk ${ }^{++}$.

\begin{tabular}{|c|c|c|c|c|}
\hline Variable & $\begin{array}{l}\text { Mbekweni - } n \text { (\%) } \\
\text { (99\% black African ancestry) }\end{array}$ & $\begin{array}{l}\text { TC Newman - } n(\%) \\
(98 \% \text { mixed ancestry) }\end{array}$ & Total - $n$ (\%) & $P$-value \\
\hline Number of mothers & $151(56)$ & $120(44)$ & $271(100)$ & 0.06 \\
\hline Primigravida & $49(32)$ & $42(35)$ & $91(34)$ & 0.76 \\
\hline Married/cohabiting & $54(36)$ & $52(43)$ & $106(39)$ & 0.27 \\
\hline Some secondary education & $87(58)$ & $54(45)$ & $141(52)$ & \\
\hline Completed secondary education & $45(30)$ & $50(42)$ & $95(35)$ & \\
\hline Tertiary education & $9(6)$ & $5(4)$ & $14(5)$ & \\
\hline Employed & $33(22)$ & $39(33)$ & $72(27)$ & 0.07 \\
\hline \multicolumn{5}{|l|}{ Estimated household income } \\
\hline HIV-infected & $58(38)$ & $6(5)$ & $64(24)$ & $<0.01$ \\
\hline \multicolumn{5}{|l|}{ Maternal antenatal psychosocial risk } \\
\hline Lifetime trauma exposure (mPSS/LEQ) & $107(71)$ & $63(53)$ & $170(63)$ & $<0.01$ \\
\hline Lifetime trauma exposure (composite) & $137(91)$ & $101(84)$ & $238(88)$ & 0.07 \\
\hline Childhood maltreatment (CTQ): above threshold & $52(34)$ & $61(51)$ & $113(42)$ & 0.01 \\
\hline \multicolumn{5}{|l|}{ Intimate Partner Violence (IPV) exposure } \\
\hline Any lifetime exposure & $69(46)$ & $76(63)$ & $145(54)$ & 0.01 \\
\hline Any recent exposure & $56(37)$ & $59(49)$ & $115(42)$ & 0.07 \\
\hline Stressful life events: median (IQR) score & $1(1 ; 3)$ & $3(1 ; 4)$ & $2(1 ; 3)$ & $<0.01$ \\
\hline Psychological distress (SRQ-20): above threshold & $39(26)$ & $39(33)$ & $78(29)$ & 0.27 \\
\hline
\end{tabular}

\footnotetext{
${ }^{++}$Prevalence computed including missing data.
}

\section{Anthropometry at birth}

More than half (55\%) of children in this study sample was male; and the median (IQR) gestational age at birth was $39(38 ; 40)$ weeks, Table 2 . Approximately a fifth $(21 \%)$ of the index deliveries were by Caesarean section. The prevalence each of preterm birth and of low birth weight was $11 \%$; and of decreased weight-for-age z (WAZ) scores at birth and low head-circumference-for-age $z$ (HCAZ) scores, $8 \%$ and $13 \%$, respectively. The median (IQR) weight at birth in this study sample was $3.1(2.8 ; 3.5) \mathrm{kg}$; and the median (IQR) head circumference was $34(33 ; 35) \mathrm{cm}$.

\section{Associations between maternal psychosocial risk and gestational epigenetic age deviation at birth}

In bivariate unadjusted analyses, mode of delivery $(\beta=1.87 ; p=$ $0.026)$, newborn head circumference at birth $(\beta=0.62 ; p=$ $0.001)$, birth weight $(\beta=1.97 ; p=0.002)$ and weight-for-age $z$ score at birth $(\beta=0.84 ; p=0.012)$, each was found to be significantly positively associated with gestational EA residuals at birth, using the conventional $p$-value $<0.05$ cut-off. When accounting for potential trend-level relationships $(p<0.1)$, positive associations with the outcomes of interest were also noted for estimated household monthly income of less that ZAR $1000(\approx$ USD 70) $(\beta=1.35 ; p=0.052)$, maternal body mass index
(BMl; $\left.\mathrm{kg} / \mathrm{m}^{2}\right)$ at enrolment $(\beta=0.10 ; p=0.068)$ and maternal anaemia $(\beta=1.60 ; p=0.091)$.

Maternal psychological distress $(\beta=-1.57 ; p=0.038)$ and low infant birthweight $(\beta=-2.33 ; p=0.035)$ were found to be significantly negatively associated with gestational EA residuals at birth; while maternal HIV $(\beta=-1.35 ; 0.094)$ and PTSD $(\beta=$ $-1.24 ; \quad p=0.096)$ showed trend-level negative associations (Table 3, Supplementary Table A).

In the final multivariable regression model, maternal PTSD was significantly and negatively associated with gestational EA residuals at birth $(\beta=-1.95 ; p=0.018)$, when controlling for study site, sex of the child, head circumference at birth, birthweight, mode of delivery, maternal estimated household income, BMI at enrolment, HIV status, anaemia, psychological distress, prenatal tobacco use and alcohol consumption, Table 3.

\section{DISCUSSION}

In this study of the association between maternal exposure to prenatal psychosocial risk factors and gestational epigenetic age (EA) deviation at birth in the index children, we found a negative association with EA residuals in children born to mothers with 


\begin{tabular}{|c|c|c|c|c|}
\hline Variable & Mbekweni - $n$ (\%) & TC Newman - $n(\%)$ & Total - $n(\%)$ & $P$-value \\
\hline \multicolumn{5}{|l|}{ Sex of child } \\
\hline Female & $70(46)$ & $51(43)$ & $121(45)$ & \\
\hline Gestational age at birth: median (IQR) weeks & $39(38 ; 40)$ & $39(38 ; 40)$ & $39(38 ; 40)$ & 0.79 \\
\hline Preterm birth & $18(12)$ & $12(10)$ & $30(11)$ & 0.76 \\
\hline Birthweight: median (IQR) kg & $3.2(2.9 ; 3.5)$ & $3.0(2.7 ; 3.4)$ & $3.1(2.8 ; 3.5)$ & 0.01 \\
\hline Low birthweight: $<2500 \mathrm{~g}$ & $14(9)$ & $15(13)$ & $29(11)$ & 0.54 \\
\hline Birth WAZ: median (IQR) & $-0.4(-1.1 ; 0.2)$ & $-0.7(-1.4 ;-0.1)$ & $-0.5(-1.3 ;-0.01)$ & 0.03 \\
\hline Low WAZ at birth: $\leq 2$ SDs below mean weight-for-age & $11(7)$ & $11(9)$ & $22(8)$ & 0.76 \\
\hline Head circumference at birth: median (IQR) cm & $34(33 ; 35)$ & $34(32 ; 35)$ & $34(33 ; 35)$ & 0.06 \\
\hline
\end{tabular}

PTSD (compared to those without such exposure), when controlling for a number of relevant covariates.

These findings are comparable to emerging-albeit mixedevidence of trauma-/PTSD-associated EA deviation in adult combat veterans. For example, recent work has demonstrated a positive association each between lifetime PTSD severity [58] and PTSD hyperarousal symptoms [59], and EA residualized for chronological age in male and female veterans (although the association with PTSD severity was not replicated in the latter study [59]). Further, a meta-analysis by the Psychiatric Genomics Consortium PTSD Epigenetics Workgroup across a number of adult cohorts reported small but significant associations each between childhood trauma exposure and severity of lifetime PTSD, and accelerated epigenetic aging (positive EA residuals) [60]. However, neither current PTSD diagnosis/severity nor lifetime trauma exposure was associated with this outcome. In addition, a small longitudinal study of male soldiers $(n=96)$, assessing preand post-deployment EA reported that the development of PTSD symptoms was associated with a relative decrease in EA over time (although trauma was found to be significantly associated with an increase in EA in this study) [61].

Work in paediatric study samples has yielded similarly varied findings. For example, one recent study $(N=101)$ reported a positive correlation between age acceleration (measured as the residual between EA and chronological age) and direct exposure to neighbourhood violence in children aged 6-13 years [62]; though-as discussed above-a negative association between distress and EA deviation has also been reported [18]. Thus, it may be that any deviation in EA (i.e., greater or lower EA relative to chronological age) is associated with exposure to prenatal maternal risk [15, 17]; and that the functional relevance and implications of accelerated epigenetic aging in infants and children are distinct from those previously demonstrated in adult study samples [12-14].

A number of limitations of the current study should be acknowledged. First, the sample size was relatively small, thus decreasing the statistical power of association analyses. Nevertheless, we report an important association with PTSD exposure even after taking into account study site, sex of the child, head circumference at birth, birthweight, mode of delivery, maternal estimated household income, BMI at enrolment, HIV status, anaemia, psychological distress, and prenatal tobacco or alcohol use. Second, self-report questionnaires to assess maternal exposure to psychosocial risk factors may have resulted in under-reporting of key variables such as substance use. Given prior evidence of associations between maternal smoking and alcohol consumption and deviations in EA [63], self-reporting bias may have contributed to the lack of significant associations in our analysis. However, we have previously shown that self-reported tobacco smoking correlated well with urine cotinine measurement (an objective biomarker of tobacco exposure) in our study cohort; particularly in the TC Newman sample [27]. Third, PTSD was not assessed using a clinician-administered diagnostic interview, which may have biased the findings. Further, heterogenous and dynamic definitions of trauma exposure and PTSD applied in the field may also limit the interpretation and generalisability of our current findings. For example, investigators may elect a "strict" approach using the DSM 5 Criterion A definition for trauma exposure [64], versus adopting a more inclusive approach, e.g., incorporating subjectively stressful events which do not necessarily meet the DSM criteria (as we have done in the current analysis). Similarly, "broad" versus "narrow" definitions of PTSD [65] would also impact estimations of case prevalence; and our findings were not replicated with more stringent definitions of trauma/PTSD.

Fourth, given that samples for analysis using the EPIC array were initially selected primarily based on maternal trauma or PTSD phenotype, the multivariable regression models could not be further stratified by chip or batch, due to the confounding effect of our selection criteria. We did, however, attempt to address potential batch effects by stratifying the age residuals by batch. Given the noted site-specific differences in maternal PTSD and other key phenotypes (e.g., tobacco use), we also included study site as a covariate in the multivariable regression model. Fifth, gestational age estimation (including by ultrasound) may be prone to notable measurement error [66-68], which may in turn have biased our primary outcome variable (gestational EA residuals at birth). Finally, inconsistencies between our results and the null findings of the well-powered epigenome-wide metaanalysis of maternal stress [6] (discussed in the Introduction, above) may be accounted for by differences in analytic approaches (e.g., differential DNAm versus EA deviation analysis); in microarray chip used (only $450 \mathrm{~K}$ data were included in the meta-analysis); in sample size; or in phenotype variables (e.g., ancestral diversity in our study sample, differing prevalence of prenatal maternal smoking and variable definitions of stress, trauma and PTSD).

These limitations notwithstanding, our study provides a unique preliminary exploration of associations between maternal psychosocial risk factors and child gestational EA deviation at birth in a high-risk LMIC environment. To the best of our knowledge, an association between maternal PTSD and gestational EA deviation 
Table 3. Associations between maternal psychosocial risk factors and gestational epigenetic age deviation at birth.

\begin{tabular}{|c|c|c|c|c|}
\hline & $\begin{array}{l}\text { Unadjusted } \\
\beta \text { estimate }[95 \% \mathrm{Cl}]\end{array}$ & $P$-value & $\begin{array}{l}\text { Adjusted } \\
\beta \text { estimate }[95 \% \mathrm{Cl}]\end{array}$ & $P$-value \\
\hline Estimated household income: < ZAR 1000/month & $1.35[-0.01 ; 2.71]$ & $0.052^{\mathrm{b}}$ & $1.87[0.40 ; 3.34]$ & $0.013^{\mathrm{a}}$ \\
\hline Mother HIV-infected & $-1.35[-2.93 ; 0.23]$ & $0.094^{b}$ & $-1.87[-3.74 ;-0.01]$ & $0.049^{\mathrm{a}}$ \\
\hline Maternal anaemia & $1.60[-0.26 ; 3.47]$ & $0.091^{\mathrm{b}}$ & $1.70[-0.24 ; 3.64]$ & $0.085^{\mathrm{b}}$ \\
\hline Child head circumference at birth & $0.62[0.25 ; 0.98]$ & $0.001^{\mathrm{a}}$ & $0.20[-0.34 ; 0.74]$ & 0.469 \\
\hline Child weight at birth & $1.97[0.73 ; 3.20]$ & $0.002^{\mathrm{a}}$ & $1.62[-0.13 ; 3.37]$ & $0.069^{b}$ \\
\hline Alcohol exposure during pregnancy & $-0.13[-1.91 ; 1.65]$ & 0.885 & $0.13[-1.92 ; 2.17]$ & 0.904 \\
\hline Tobacco use during pregnancy & $-0.76[-2.23 ; 0.72]$ & 0.313 & $-0.20[-2.19 ; 1.79]$ & 0.843 \\
\hline Psychological distress & $-1.57[-3.04 ;-0.09]$ & $0.038^{\mathrm{a}}$ & $-0.76[-2.40 ; 0.88]$ & 0.363 \\
\hline
\end{tabular}

${ }^{a} p<0.05$.

$\mathrm{b}_{p}<0.1$.

'BMI (Body Mass Index): $\mathrm{kg} / \mathrm{m}^{2}$.

in the index children has not previously been reported. Future studies-incorporating larger samples sizes and cross-tissue analyses-are warranted in order to explore such associations further; and to delineate underlying neurobiological mechanisms. In addition, work is needed to understand the implications of EA deviation at birth. For example, it is currently unknown whether and how EA deviation may also be associated with adverse neurodevelopmental outcomes in the affected children, although interest in this field is growing [17]. Further, while the adverse transgenerational effects of maternal trauma exposure and PTSD have been well documented-both in the DCHS cohort [23-25] and globally $[1,69]$ - the potential role of EA deviation in these associations is yet to be determined. From a translational perspective, if EA deviation at birth does indeed prove clinically meaningful, it may contribute to an improved neurobiological understanding of transgenerational trauma and PTSD, which may in turn inform the development of epigenetic biomarkers in this context.

\section{REFERENCES}

1. Shah PS, Shah J. Knowledge Synthesis Group on Determinants of Preterm/LBW Births. Maternal exposure to domestic violence and pregnancy and birth outcomes: a systematic review and meta-analyses. J. Women's Health (Larchmt.). 2010;19:2017-1231.

2. Grigoriadis S, Graves L, Peer M, Mamisashvili L, Tomlinson G, Vigod SN, et al. Maternal anxiety during pregnancy and the association with adverse perinatal outcomes: systematic review and meta-analysis. J. Clin. Psychiatry. 2018;4:79. 17r12011

3. Testa M, Quigley BM, Eiden RD. The effects of prenatal alcohol exposure on infant mental development: a meta-analytical review. Alcohol Alcohol. 2003;38:295-304.

4. Atwoli L, Stein DJ, Williams DR, Mclaughlin KA, Petukhova M, Kessler RC, et al. Trauma and posttraumatic stress disorder in South Africa: analysis from the South African Stress and Health Study. BMC Psychiatry. 2013;13:182.

5. Palma-Gudiel H, Córdova-Palomera A, Eixarch E, Deuschle M, Fañanás L. Maternal psychosocial stress during pregnancy alters the epigenetic signature of the glucocorticoid receptor gene promoter in their offspring: a meta-analysis. Epigenetics. 2015;10:893-902.

6. Rijlaarsdam J, Pappa I, Walton E, Bakermans-Kranenburg MJ, Mileva-Seitz VR, Rippe $\mathrm{RC}$, et al. An epigenome-wide association meta-analysis of prenatal maternal stress in neonates: a model approach for replication. Epigenetics. 2016;11:140-9.

7. Horvath S. DNA methylation age of human tissues and cell types. Genome Biol. 2013;14:R115.

8. Hannum G, Guinney J, Zhao L, Zhang L, Hughes G, Sadda S, et al. Genome-wide methylation profiles reveal quantitative views of human aging rates. Mol. Cell. 2013;49:359-67.
9. Knight AK, Craig JM, Theda C, Bækvad-Hansen M, Bybjerg-Grauholm J, Hansen $\mathrm{CS}$, et al. An epigenetic clock for gestational age at birth based on blood methylation data. Genome Biol. 2016;17:206.

10. Knight AK, Conneely KN, Smith AK Gestational age predicted by DNA methylation: potential clinical and research utility. Epigenomics 2017; Jan. https://doi.org/ 10.2217/epi-2016-0157. [Epub ahead of print].

11. Bohlin J, Håberg SE, Magnus P, Reese SE, Gjessing HK, Magnus MC, et al. Prediction of gestational age based on genome-wide differentially methylated regions. Genome Biol. 2016;17:207.

12. Chen BH, Marioni RE, Colicino E, Peters MJ, Ward-Caviness CK, Tsai PC, et al. DNA methylation-based measures of biological age: meta-analysis predicting time to death. Aging (Albany, NY). 2016;8:1844-65.

13. Marioni RE, Shah S, McRae AF, Chen BH, Colicino E, Harris SE, et al. DNA methylation age of blood predicts all-cause mortality in later life. Genome Biol. 2015;16:25.

14. Fransquet PD, Wrigglesworth J, Woods RL, Ernst ME, Ryan J. The epigenetic clock as a predictor of disease and mortality risk: a systematic review and metaanalysis. Clin. Epigenetics. 2019;11:62.

15. Girchenko P, Lahti J, Czamara D, Knight AK, Jones MJ, Suarez A, et al. Associations between maternal risk factors of adverse pregnancy and birth outcomes and the offspring epigenetic clock of gestational age at birth. Clin Epigenetics. 2017;9:49. eCollection 2017

16. Khouja JN, Simpkin AJ, O'Keeffe LM, Wade KH, Houtepen LC, Relton $C L$, et al. Epigenetic gestational age acceleration: a prospective cohort study investigating associations with familial, sociodemographic and birth characteristics. Clin Epigenetics. 2018;10:86.

17. Suarez A, Lahti J, Czamara D, Lahti-Pulkkinen M, Knight AK, Girchenko $P$, et al. The epigenetic clock at birth: associations with maternal antenatal depression and child psychiatric problems. J Am Acad Child Adolesc Psychiatry. 2018;57:321-8.

18. Moore SR, McEwen LM, Quirt J, Morin A, Mah SM, Barr RG, et al. Epigenetic correlates of neonatal contact in humans. Dev Psychopathol. 2017;29:1517-38.

19. Zar HJ, Barnett W, Myer L, Stein DJ, Nicol MP. Investigating the early-life determinants of illness in Africa: the Drakenstein Child Health Study. Thorax. 2015;70:592-4.

20. Stein DJ, Koen N, Donald KA, Adnams CM, Koopowitz S, Lund C, et al. Investigating the psychosocial determinants of child health in Africa: The Drakenstein Child Health Study. J Neurosci Methods. 2015;252:27-35.

21. Donald KA, Hoogenhout M, du Plooy CP, Wedderburn CJ, Nhapi RT, Barnett W, et al. Drakenstein Child Health Study (DCHS): investigating determinants of early child development and cognition. BMJ Paediatr Open. 2018;2:e000282. eCollection 2018

22. Zar HJ, Pellowski JA, Cohen S, Barnett W, Vanker A, Koen N, et al. Maternal health and birth outcomes in a South African birth cohort study. PLoS ONE. 2019;14: e0222399. eCollection 2019

23. Koen N, Wyatt GE, Williams JK, Zhang M, Myer L, Zar HJ, et al. Intimate partner violence: associations with low infant birthweight in a South African birth cohort. Metab. Brain Dis. 2014;29:281-99. 
24. Koen N, Brittain K, Donald KA, Barnett W, Koopowitz S, Maré K, et al. Psychological trauma and posttraumatic stress disorder: risk factors and associations with birth outcomes in the Drakenstein Child Health Study. Eur J Psychotraumatol. 2016;7:28720. eCollection 2016

25. Koen N, Brittain K, Donald KA, Barnett W, Koopowitz S, Maré K, et al. Maternal posttraumatic stress disorder and infant developmental outcomes in a South African birth cohort study. Psychol Trauma. 2017;9:292-300.

26. Myers B, Koen N, Donald KA, Nhapi RT, Workman L, Barnett W, et al. Effect of hazardous alcohol use during pregnancy on growth outcomes at birth: findings from a South African cohort study. Alcohol Clin Exp Res. 2018;42:369-77.

27. Vanker A, Barnett W, Brittain K, Gie RP, Koen N, Myers B, et al. Antenatal and early life tobacco smoke exposure in an African birth cohort study. Int J Tuberc Lung Dis. 2016;20:729-37.

28. Morin AM, Gatev E, McEwen LM, Maclsaac JL, Lin D, Koen N, et al. Maternal blood contamination of collected cord blood can be identified using DNA methylation at three CpGs. Clin Epigenetics. 2017;9:75.

29. Myer L, Stein DJ, Grimsrud A, Seedat S, Williams DR. Social determinants of psychological distress in a nationally-representative sample of South African adults. Soc Sci Med. 2008;66:1828-40.

30. Bernstein DP, Fink L, Handelsman L, Foote J, Lovejoy M, Wenzel K, et al. Initial reliability and validity of a new retrospective measure of child abuse and neglect. Am J Psychiatry. 1994;151:1132-6.

31. García-Moreno C, Jansen HAFM, Ellsberg M, Heise L, Watts C (2005) WHO multicountry study on women's health and domestic violence against women: Initial results on prevalence, health outcomes and women's responses. World Health Organization. Available at: https://www.who.int/reproductivehealth/publications/ violence/24159358X/en/ [Accessed 19 February 2020].

32. Foa EB, Riggs DS, Dancu CV, Rothbaum BO. Reliability and validity of a brief instrument for assessing posttraumatic stress disorder. J. Trauma Stress. 1993;6:459-73.

33. Falsetti SA, Resnick HS, Resick PA, Kilpatrick D. The Modified PTSD Symptom Scale: A brief self-report measure of posttraumatic stress disorder. Behav Therapist. 1993;16:161-2.

34. Lecrubier $\mathrm{Y}$, Sheehan $\mathrm{D}$, Weiller $\mathrm{E}$, Amorim $\mathrm{P}$, Bonora I, Sheehan $\mathrm{KH}$, et al. The MINI International Neuropsychiatric Interview (M.I.N.I.) a short diagnostic structured interview: reliability and validity according to the CIDI. Eur Psychiatry. 1997;12:224-31

35. Sheehan DV, Lecrubier $Y$, Sheehan $K H_{\text {, Amorim }} \mathrm{P}$, Janavs J, Weiller $\mathrm{E}$, et al. The Mini-International Neuropsychiatric Interview (M.I.N.I.): the development and validation of a structured diagnostic psychiatric interview for DSMIV and ICD-10. J Clin Psychiatry. 1998;59:22-33.

36. Sheehan D, Lecrubier Y, Harnett Sheehan K, Janavs J, Weiller E, Keskiner A, et al. The validity of the Mini International Neuropsychiatric Interview (MINI) according to the SCID-P and its reliability. Eur Psychiatry. 1997;12:232-41.

37. Harding TW, de Arango MV, Baltazar J, Climent CE, Ibrahim HH, Ladrido-Ignacio L, et al. Mental disorders in primary health care: a study of their frequency and diagnosis in four developing countries. Psychol Med. 1980;10:231-41.

38. Scholte WF, Verduin F, van Lammeren A, Rutayisire T, Kamperman AM. Psychometric properties and longitudinal validation of the self-reporting questionnaire (SRQ-20) in a Rwandan community setting: a validation study. BMC Med Res Methodol. 2011;11:116. https://doi.org/10.1186/1471-2288-11-116

39. van der Westhuizen C, Wyatt G, Williams JK, Stein DJ, Sorsdahl K. Validation of the Self Reporting Questionnaire 20-item (SRQ-20) for use in a low- and middleincome country emergency centre setting. Int J Ment Health Addict. 2016;14:37-48.

40. Beck AT, Ward CH, Mendelson M, Mock J, Erbaugh J. An inventory for measuring depression. Arch Gen Psychiatry. 1961;4:53-63.

41. Beck AT, Steer RA, Garbin MG. Psychometric properties of the Beck Depression Inventory: twenty five years of evaluation. Clin Psychol Rev. 1988;8:77-100.

42. Beck AT, Steer RA, Brown GK Manual for the Beck Depression Inventory-II. Psychological Corporation: San Antonio, TX, 1996.

43. Cox JL, Holden JM, Sagovsky R. Detection of postnatal depression: development of the 10-item Edinburgh Postnatal Depression Scale. Br J Psychother. 1987;150:782-6.

44. Eberhard-Gran M, Eskild A, Tambs K, Opjordsmoen S, Samuelsen SO. Review of validation studies of the Edinburgh Postnatal Depression Scale. Acta Psychiatr Scand. 2001;104:243-9.

45. WHO ASSIST Working Group. The Alcohol, Smoking and Substance Involvement Screening Test (ASSIST): development, reliability and feasibility. Addiction. 2002;97:1183-94.

46. Humeniuk R, Ali R, Babor TF, Farrell M, Formigoni ML, Jittiwutikarn J, et al. Validation of the alcohol, smoking and substance involvement screening test (ASSIST). Addiction. 2008;103:1039-47.

47. van der Westhuizen C, Wyatt G, Williams J, Stein D, Sorsdahl K. Validation of the Alcohol, Smoking and Substance Involvement Screening Test in a low- and middle-income country cross-sectional emergency centre study. Drug Alcohol Rev. 2016;35:702-9.
48. Fenton TR. A new growth chart for preterm babies: Babson and Benda's chart updated with recent data and a new format. BMC Pediatr. 2003;3:13.

49. Fenton TR, Kim JH. A systematic review and meta-analysis to revise the Fenton growth chart for preterm infants. BMC Pediatr. 2013;13:59.

50. World Health Organization (WHO) Physical status: the use and interpretation of anthropometry. Report of a WHO Expert Committee. World Health Organ Tech. Rep Ser. 1995;854:1-452.

51. R Core Team. R: A Language and Environment for Statistical Computing. R Foundation for Statistical Computing, 2017. https://www.R-project.org/

52. StataCorp. Stata Statistical Software: Release 14. College Station. StataCorp LP, 2015.

53. Moran S, Arribas C, Esteller M. Validation of a DNA methylation microarray for $850,000 \mathrm{CpG}$ sites of the human genome enriched in enhancer sequences. Epigenomics. 2016;8:389-99.

54. Czamara D, Eraslan G, Page CM, Lahti J, Lahti-Pulkkinen $M$, Hämäläinen $E$, et al. Integrated analysis of environmental and genetic influences on cord blood DNA methylation in newborns. Nat. Commun. 2019;10:2548.

55. Magnus $P$, Irgens LM, Haug K, Nystad W, Skjaerven R, Stoltenberg C, MoBa Study Group.Cohort profile: the Norwegian Mother and Child Cohort Study (MoBa). Int Epidemiol. 2006;35:1146-50.

56. Magnus P, Birke C, Vejrup K, Haugan A, Alsaker E, Daltveit AK, et al. Cohort profile update: The Norwegian Mother and Child Cohort Study (MoBa). Int J Epidemiol. 2016;45:382-8

57. McEwen LM, Jones MJ, Lin D, Edgar RD, Husquin LT, Maclsaac JL, et al. Systematic evaluation of DNA methylation age estimation with common preprocessing methods and the Infinium MethylationEPIC BeadChip array. Clin Epigenetics. 2018;10:123. https://doi.org/10.1186/s13148-018-0556-2

58. Wolf EJ, Logue MW, Hayes JP, Sadeh N, Schichman SA, Stone A, et al. Accelerated DNA methylation age: Associations with PTSD and neural integrity. Psychoneuroendocrinology. 2016;63:155-62.

59. Wolf EJ, Logue MW, Stoop TB, Schichman SA, Stone A, Sadeh N, et al. Accelerated DNA methylation age: Associations with posttraumatic stress disorder and mortality. Psychosom Med. 2018;80:42-48.

60. Wolf EJ, Maniates H, Nugent N, Maihofer AX, Armstrong D, Ratanatharathorn A et al. Traumatic stress and accelerated DNA methylation age: a meta-analysis. Psychoneuroendocrinology. 2018;92:123-34.

61. Boks MP, van Mierlo HC, Rutten BP, Radstake TR, De Witte L, Geuze E, et al. Longitudinal changes of telomere length and epigenetic age related to traumatic stress and post-traumatic stress disorder. Psychoneuroendocrinology. 2015;51:506-12.

62. Jovanovic T, Vance LA, Cross D, Knight AK, Kilaru V, Michopoulos V, et al. Exposure to violence accelerates epigenetic aging in children. Sci Rep. 2017;7:8962. https://doi.org/10.1038/s41598-017-09235-9

63. Simpkin AJ, Hemani G, Suderman M, Gaunt TR, Lyttleton O, Mcardle WL, et al. Prenatal and early life influences on epigenetic age in children: a study of motheroffspring pairs from two cohort studies. Hum Mol Genet. 2016;25:191-201.

64. American Psychiatric Association (APA). Diagnostic and Statistical Manual of Mental Disorders, $5^{\text {th }}$ Edition. American Psychiatric Publishing: Arlington, VA, 2013.

65. Stein DJ, McLaughlin KA, Koenen KC, Atwoli L, Friedman MJ, Hill ED, et al. DSM-5 and ICD-11 definitions of posttraumatic stress disorder: investigating "narrow" and "broad" approaches. Depress Anxiety. 2014;31:494-505.

66. Lynch CD, Zhang J. The research implications of the selection of a gestational age estimation method. Paediatr Perinat Epidemiol. 2007;21:86-96.

67. Geerts L, Poggenpoel E, Theron G. A comparison of pregnancy dating methods commonly used in South Africa: a prospective study. S Afr Med J. 2013;103:552-6.

68. Skalkidou A, Kullinger M, Georgakis MK, Kieler H, Kesmodel US. Systematic misclassification of gestational age by ultrasound biometry: implications for clinical practice and research methodology in the Nordic countries. Acta Obstet Gynecol Scand. 2018:97:440-4.

69. Roberts AL, Galea S, Austin SB, Cerda M, Wright RJ, Rich-Edwards JW, et al. Posttraumatic stress disorder across two generations: concordance and mechanisms in a population-based sample. Biol Psychiatry. 2012;72:505-11.

\section{ACKNOWLEDGEMENTS}

This study was supported by the Eunice Kennedy Shriver National Institute of Child Health and Human Development of the National Institutes of Health (NICHD) under Award Number R21HD085849, the Fogarty International Center (FIC), and the Bill and Melinda Gates Foundation (OPP 1017641). The content is solely the responsibility of the authors and does not necessarily represent the official views of the National Institutes of Health. Additional support for HJZ, DJS, NK, and WB, and for research reported in this publication was by the South African Medical Research Council (SAMRC); from a Newton Advanced Fellowship (KD); and from South Africa's National Research Foundation (NRF) (HZ; grant number: 105865). WB is supported by the SAMRC National Health Scholars programme through its Division of Research Capacity Development; NK and KD received additional support from the SAMRC under Self-Initiated Research Grants. The views and opinions expressed are those of the authors and do not necessarily represent 


\section{8}

the official views of the SAMRC. We thank the Drakenstein Child Health Study staff, and the clinical and administrative staff of the Western Cape Government Department of Health at Paarl Hospital and at the clinics for support of the study. We also thank Lesley Workman for additional data management support for this analysis. We thank our collaborators and students. Finally, we thank all mothers and children enroled in the Drakenstein Child Health Study.

\section{COMPETING INTERESTS}

The authors declare no competing interests.

\section{ADDITIONAL INFORMATION}

Supplementary information The online version contains supplementary material available at https://doi.org/10.1038/s41398-021-01434-3.

Correspondence and requests for materials should be addressed to N.K.

Reprints and permission information is available at http://www.nature.com/reprints
Publisher's note Springer Nature remains neutral with regard to jurisdictional claims in published maps and institutional affiliations.

(c) (i)

Open Access This article is licensed under a Creative Commons Attribution 4.0 International License, which permits use, sharing, adaptation, distribution and reproduction in any medium or format, as long as you give appropriate credit to the original author(s) and the source, provide a link to the Creative Commons license, and indicate if changes were made. The images or other third party material in this article are included in the article's Creative Commons license, unless indicated otherwise in a credit line to the material. If material is not included in the article's Creative Commons license and your intended use is not permitted by statutory regulation or exceeds the permitted use, you will need to obtain permission directly from the copyright holder. To view a copy of this license, visit http://creativecommons. org/licenses/by/4.0/.

(c) The Author(s) 2021 